\title{
SETS OF FORMULAS VALID IN FINITE STRUCTURES $\left({ }^{1}\right)$
}

BY

\author{
ALAN L. SELMAN
}

ABSTRACT. A function $O$ is defined on the set of all subsets of $\omega$ so that for each set $K$, the value, ${ }{ }_{K}$, is the set of formulas valid in all structures of cardinality in $K$. An analysis is made of the dependence of $\theta_{K}$ on $K$. For any set $K$, let $\mathrm{d}(K)$ be the Kleene-Post degree to which $K$ belongs. It is easily seen that for all infinite sets $K, \mathrm{~d}(K) \vee 1 \leq \mathrm{d}\left(\theta_{K}\right) \leq \mathrm{d}(K)^{\prime}$. On the other hand, we prove that $d\left(\vartheta_{K \vee J}\right)=d\left(\mho_{K}\right) \vee d\left(\vartheta_{J}\right)$, and use this to prove that, for any two degrees $a$ and $b, a \geq 1, a \leq b \leq a^{\prime}$, and $b$ r.e. $a$, there exists a set $K$ so that $\mathrm{d}(K)=a$ and $\mathrm{d}\left(\mathcal{O}_{K}\right)=\mathrm{b}$. Various similar results are also included.

B. A. Trachtenbrot [10] has shown that the set of formulas of first order logic valid in all finite structures is not recursively enumerable, although it is the complement of such a set. Let us define a function $\mathcal{O}$ on the set of all subsets of $\omega$ so that for each set $K$, the value, $\bigcup_{K}$, is the set of formulas valid in all structures of cardinality in K. A. Mostwoski has asked (in conversation, 1966) what can be said of $\mathcal{O}_{K}$, when $K$ is known. In particular, if the Kleene-Post degrees of the two sets $K$ and $J$ are identical, are the degrees of $\vartheta_{K}$ and $\vartheta_{J}$ identical?

Let $\bar{K}$ denote the complement of the set $K$. (The universe of discourse is $\omega$ throughout.) For any set $K$, let $\mathrm{d}(K)$ be the Kleene-Post degree to which $K$ belongs. It is shown that for all infinite sets $K, \mathbf{d}(K) \bigvee \mathbf{l} \leq \mathbf{d}\left(\mho_{K}\right) \leq \mathbf{d}(K)^{\prime}$. Nevertheless, in $\S 3$ it is shown that there exist sets $K$ for which $\mathbf{d}\left(\mathcal{O}_{K}\right) \neq \mathbf{d}\left(\mathcal{O}_{\bar{K}}\right)$. This solves the above question in the negative. In $\$ 4$ we describe the extent to which $d\left(\mho_{K}\right)$ is independent from $d(K)$. The principal result in this direction is Theorem 12. The techniques used to obtain our results involve both the writing of explicit algorithms and the application of standard theorems about the degrees of unsolvability.

Presented to the Society, January 23, 1970 under the title First order formulas true in structures of arbitrary finite cardinality; received by the editors November 6, 1970 and, in revised form, June $16,1972$.

AMS (MOS) subject classifications (1970)。 Primary 02B 10, 02F 30, 02H13.

Key words and phrases. First order formulas, finite structures, $K$-representation, spectrum, spectral functions.

(1) This paper is part of the author's doctoral dissertation directed by Professor Paul Axt and partially supported by NSF Grant GP7077. 
It is assumed that we have at our disposal some first order language, $\mathfrak{\&}$, with equality whose grammar contains an infinite list of $k$-ary predicate letters $M_{i}^{k}, F_{i}^{k}$, $\ldots, i \geq 1$, for each $k . \phi, \psi, \ldots$ shall denote formulas of this language. $\phi\left(M_{1}^{1}, \ldots, M_{n}^{1}, F^{1}, \ldots\right), n \geq 1$, is a formula containing among its predicate letters one or more occurrences of the one-place predicates $M_{1}^{1}, \ldots, M_{n}^{1}, F^{1}$.

Let $\mathcal{U}$ be an interpretation of the formula $\phi$. $\mathcal{U}$ is a structure with domain $A$ and $k$-ary relations $M_{i \ell}^{k}$ corresponding to predicate letters $M_{i}^{k}$ occurring in $\phi$. We write $\|A\|$, for the cardinality of a set $A$. By the cardinality of a structure of we mean the cardinality of its domain. A structure $\mathscr{Q}$ is finite, if its domain is. We write $F_{\mathfrak{a}} \phi$, if $\phi$ is valid in $\mathcal{U}$.

Also, we will use the notation " $A \leq_{r} B$ " for " $A$ recursive in $B$ " and " $A \leq_{m}$ $B$ " for " $A$ is many-one reducible to $B$ ".

Definition 1. Let $K \subseteq \omega$.

(i) $\mathcal{O}_{K}=\left\{\phi: \forall \mathscr{Q}\left[\|A\| \in K \rightarrow F_{\mathfrak{g}} \phi\right]\right\}$;

(ii) $\mathcal{W}_{K}=\left\{\phi: \quad \exists \mathscr{Q}\left[F_{y} \phi \&\|A\| \in K\right]\right\}$;

(iii) $\mathbb{M}_{K}=\left\{\phi: \forall \mathscr{Q}\left[\left(\vDash_{\mathfrak{q}} \phi \&\|A\|<\omega\right) \rightarrow\|A\| \in K\right]\right\}$.

Lemma 1. $\phi \in \mathbb{M}_{K}$ iff $\phi \notin \mathbb{C}_{\bar{K}} . \phi \in \mathcal{O}_{K}$ iff $\phi \notin \mathfrak{C O}_{K}$.

$\mathcal{W}_{K}$ and $\mathfrak{M}_{K}$ defined above have conceptual interest, and, by Lemma 1 , for each set $K, d\left(\vartheta_{K}\right)=\mathbf{d}\left(\mathcal{M}_{K}\right)=\mathbf{d}\left(\mathcal{W}_{K}\right)$. In fact, we prefer to analyze the function 60 , since as is easily seen, for all $K, W_{K}$ is r.e. in $K$.

1. Trachtenbrot's theorem. This section is concerned with certain generalizations of Theorem 1 of [10].

Throughout this paper we equate computable with recursive. As an instance, given a formula $\phi\left(M_{1}^{1}, \ldots, M_{n}^{1}, F^{1}, \ldots\right), n \geq 1$, define $\rho\left(m_{1}, \ldots, m_{n}, j, k\right)$ to be 0 if $\phi$ has a model $\mathcal{U}$ of cardinality $k$ so that $\left\|M_{i \mathfrak{V}}^{1}\right\|=m_{i}$, for $i \leq n$, and $\left\|F_{\mathfrak{q}}^{1}\right\|$ $=j$, and 1 otherwise. $\rho$ is recursive.

Definition 2. Let $K$ be a nonempty subset of $\omega$. A formula $\phi\left(M_{1}^{1}, \ldots, M_{n}^{1}, F^{1}, \ldots\right)$ is a $K$-representation of an $n$-place function $f$ if

(i) $\forall m_{1}, \cdots, m_{n}, \exists j, k\left\lfloor\rho\left(m_{1}, \ldots, m_{n}, j, k\right)=0 \& k \in K\right\rfloor$, and

(ii) $\forall k, m_{1}, \ldots, m_{n}, j\left[k \in K \& \rho\left(m_{1}, \cdots, m_{n}, i, k\right)=0 \rightarrow f\left(m_{1}, \cdots, m_{n}\right)=j\right]$.

The proof of the following theorem is immediate.

Theorem 1. If $\phi\left(M_{1}^{1}, \ldots, M_{n}^{1}, F^{1}, \ldots\right)$ is a $K$-representation of $f$, then

$$
f\left(m_{1}, \ldots, m_{n}\right)=j \leftrightarrow \exists k\left[k \in K \& \rho\left(m_{1}, \ldots, m_{n}, j, k\right)=0\right] .
$$

Theorem 2. If $f$ has a $K$-representation, then $f$ is recursive in $K$. If $f$ bas a $K$-representation and $K$ is r.e. in a set $B$, then $f$ is recursive in $B$. 
Proof. If $f$ has a $K$-representation, then, using Theorem 1 , the graph of $f$ is r.e. in any set $B$ in which $K$ is r.e. So $f$ is recursive in $K$, and if $K$ is r.e. in $B$, then $f$ is recursive in $B$.

Corollary 1. If $f$ has an $\omega$-representation, then $f$ is recursive.

Corollary 1 is due to Trachtenbrot.

Theorem 3. If $B$ is an infinite set and $f$ is recursive, then $f$ has a B-representation.

Proof. The proof is essentially a repetition of the proof of Theorem 1 in [10]. It is shown in [10] that for each recursive function $f$ there is an $\omega$-representation $\phi$. To complete the proof, it suffices to observe for each $\omega$-representation $\phi$, that if $\mathcal{O}$ is a model of $\phi$ with domain $A$, and if $\mathcal{U}$ is extended to a structure $\mathfrak{O}^{\prime}$ simply by enlarging the domain $A$, then $\mathcal{U}^{\prime}$ is a model of $\phi$. Since $B$ is an infinite set, each $\omega$-representation $\phi$ has a model of cardinality in $B$. Thus, $\phi$ is a $B$-representation.

Definition 3. The spectrum $S(\phi)$ of a first order formula $\phi$ is the set of all natural numbers $n$ for which $\phi$ has a model of cardinality $n$.

It is well known [1] that each $S(\phi)$ is an elementary set. (More recent results on the interesting problem of characterizing spectra may be found in [2] and [4].)

Let rng $f$ denote the range of a function $f$.

Definition 4. The class of spectral functions of $n$-arguments, $\mathrm{Spr}_{n}=$ $\left\{f: f \in \omega^{\left(\omega^{n}\right)} \& f\right.$ has an $\omega$-representation $\phi\left(M_{1}^{1}, \ldots, M_{n}^{1}, F^{1}, \ldots\right)$ so that $\operatorname{rng} f=$ $\left.S\left(\phi\left(M_{1}^{1}, \ldots, M_{n}^{1}, F^{1}, \ldots\right) \wedge \forall x F^{1} x\right)\right\}$.

Lemma 2. (1) The functions $2 x, 2 x+1$, and $x^{2}$ belong to $\mathrm{Spr}_{1}$. The function $u+x$ belongs to $\mathrm{Spr}_{2}$.

(2) $\mathrm{Spr}_{1}$ is closed under substitution. More generally, if $g \in \mathrm{Spr}_{m}$ and $f_{1}, \ldots$, $f_{m} \in \mathrm{Spr}_{n}, n, m>0$, then the function $b$ defined by

$$
b\left(x_{1}, \ldots, x_{n}\right)=g\left(f_{1}\left(x_{1}, \ldots, x_{n}\right), \ldots, f_{m}\left(x_{1}, \ldots, x_{n}\right)\right)
$$

is contained in $\mathrm{Spr}_{n}$.

Proof. (1) We again cite [10]. By that paper, the functions listed in (1) all have $\omega$-representations. It is easy to see that these representations have the required property.

(2) Let $f$ and $g$ belong to $\operatorname{Spr}_{1}$. Define $b(x)=g(f(x))$. $f$ has $\omega$-representation $\phi\left(M^{1}, F^{1}, \ldots\right)$ and $g$ has $\omega$-representation $\psi\left(M^{1}, F^{1}, \ldots\right)$, both satisfying Definition 4. By [10], $b$ has $\omega$-representation $\phi\left(M^{1}, G^{1}, \ldots\right) \wedge \psi\left(G^{1}, F^{1}, \ldots\right)$. Suppose $y=b(x)$, for some $x . \phi\left(M^{1}, G^{1}, \ldots\right)$ has a model 20 of cardinality $\left\|G_{\mathfrak{q}}^{1}\right\|=f(x)$. Extend (enlarge the domain) and expand (add additional relations) QU to a model $B$ of $\psi\left(G^{1}, F^{1}, \ldots\right)$ of cardinality $\left\|F_{\mathscr{B}}^{1}\right\|=g(f(x))=y$. As observed 
in the proof of Theorem 3, $\mathcal{Q}$ can be extended to $B$ so that $B$ is still a model of $\phi\left(M^{1}, G^{1}, \ldots\right)$. Thus, $y \in S\left(\phi\left(M^{1}, G^{1}, \ldots\right) \wedge \psi\left(G^{1}, F^{1}, \ldots\right) \wedge \forall x F^{1} x\right)$. It is immediate that if $y \in S\left(\phi\left(M^{1}, G^{1}, \ldots\right) \wedge \psi\left(G^{1}, F^{1}, \ldots\right) \wedge \forall x F^{1} x\right)$, then $y \epsilon$ rng $b$. Thus $b \in \mathrm{Spr}_{1}$.

The proof of the second statement in (2) is identical.

2. Elementary properties of 20 . Suppose a Gödel numbering is given for the set of formulas of $\mathfrak{Q}$ so that each number is used exactly once. Throughout this paper let $R(x, k)$ be the number theoretic predicate $R(x, k) \equiv$ formula with Gödel number $x$ has a model of cardinality $k . R$ is a recursive predicate. Let $\lceil\bar{\phi}$ denote the Gödel number of $\phi$, and let $\lfloor$ $x$ denote the formula with Gödel number $x$. For each set $K, \bigcup_{K}=\{\phi: \exists k(R(\ulcorner\urcorner, x) \& k \in K)\}$. But, in what follows we will instead denote $\{x \mid \exists k(R(x, k) \& k \in K)\}$ by $\mathbb{O}_{K}$.

Theorem 4. (1) For each set $K, W_{K}$ r.e. $K$. In fact, $\forall B\left[K \in \Sigma_{1}^{B} \rightarrow W_{K} \in \Sigma_{1}^{B}\right]$.

(2) If $K$ is finite, then $60_{K}$ is recursive.

(3) $K \in \Sigma_{n} \rightarrow 6_{K} \in \Sigma_{n}$.

(4) $K \in \Pi_{n}^{n} \rightarrow 0_{K} \in \Sigma_{n+1}$.

(5) For each set $K, K \leq_{m} 6 W_{K}$.

Proof. The proofs of the first four clauses are immediate. Let $E_{n}$ be a first order formula asserting the existence of exactly $n$ distinct elements. $x \in K \rightarrow$ $r_{E}^{\urcorner} \in \mathbb{C O}_{K}$. Thus, $K \leq_{m}{ } \mathrm{O}_{K}$.

If $\phi$ is a formula with one free variable, let $\exists !_{n} x \phi$ be the formula asserting that there are exactly $n$ distinct elements which satisfy $\phi$.

Theorem 5. If every function recursive in $K$ bas a K-representation, then $60_{K}$ is a completion of $K$. Thus, $\mathrm{d}\left(6_{K}\right)=\mathrm{d}(K)$.

Proof. By Theorem $4, W_{K}$ r.e. K. Suppose $P(x)$ r.e. $K . P(x) \equiv \exists k\left[f^{K}(k)=x\right]$, where $f^{K}$ is some function recursive in $K$. By assumption $f^{K}$ has a $K$-representation, say $\phi\left(M^{1}, F^{1}, \ldots\right)$. Let $g(n)$ be the number theoretic function defined by

Then

$$
\left.g(n)=r_{\phi}\left(M^{1}, F^{1}, \ldots\right) \wedge \exists !_{n} y F^{1}(y)\right\urcorner^{\circ} .
$$

$$
P(n) \equiv \exists k\left[f^{K}(k)=n\right] \equiv \exists k[R(g(n), k) \& k \in K]
$$

That is, $P(x) \leq{ }_{m} 68_{K}$. Thus, $60_{K}$ is a completion of $K$.

Corollary 2. If $f$ bas a $K$-representation, then rng $f \leq_{m} W_{K}$.

If $K$ is an infinite set, then, by Theorem 3, every recursive function has a $K$ representation. Hence, the following Corollary 3 follows from Corollary 2. 
Corollary 3. (1) $\mathrm{d}\left(\mathfrak{W}_{\omega}\right)=1$. In fact, $\mathfrak{W}_{\omega}$ is a complete $\Sigma_{1}$-set $[10$, Theorem 2]. (2) If $K$ is infinite, then $\mathbf{d}\left(6_{K}\right) \geq 1$.

Suppose $K \in \Sigma_{n+1}$. Then $k=$ rng $f$, where $f$ is recursive in $\Sigma_{n}$. That is $K$ is r.e. in a $\Sigma_{n}$-set. Thus, by Theorem 2 , if $g$ is a function with a $K$-representation, then $g$ is recursive in a $\Sigma_{n}$-set. Thus, if $K \in \Sigma_{n+1}$ and $g$ has a $K$-representation, then $g \in \Delta_{n+1}$. Hence, not every function recursive in $K$ has a $K$-representation.

This same conclusion follows from Theorem 5, since $K \in \Sigma_{n}$ implies $W_{K} \in \Sigma_{n}$.

Theorem 6. $\exists B\left[A=B^{\prime}\right] \rightarrow \mathrm{d}\left(6_{A}\right)=\mathrm{d}(A)$.

Proof. $A \in \Sigma_{1}^{B}$. Thus, $W_{A} \in \Sigma_{1}^{B}$, by Theorem 4(1). Hence, $W_{A} \leq_{r} A$, since $A=$ $B^{\prime}$. On the other hand, by Theorem 4(5), $A \leq_{m} 6{ }_{A}$.

The following corollary follows from Theorem 4 and Corollary 3.

Corollary 4. For all infinite sets $K, \mathrm{~d}(K) \bigvee \mathbf{l} \leq \mathrm{d}\left(\left(_{K}\right) \leq \mathrm{d}(K)^{\prime}\right.$.

Corollary 3 and the following examples show that Corollary 4 gives the best possible upper and lower bounds to $\mathbf{d}\left(\mathbb{W}_{K}\right)$. By Example $3, \mathrm{~d}(K)$ and $\mathbf{d}(K)^{\prime}$ are not the only possible values for $\mathrm{d}\left(6_{K}\right)$.

Examples. 1. By Theorem 6 and Friedberg's characterization of the degrees greater than $0^{\prime}[3]$,

$$
\forall \mathbf{d} \geq \mathbf{0}^{\prime} \quad \exists K\left[\mathbf{d}(K)=\mathbf{d} \& \mathbf{d}\left(\mathbb{C}_{K}\right)=\mathbf{d}(K)\right] .
$$

2. Also by the result in [3], given $\mathbf{a}>\mathbf{0}^{\prime}$, choose $\mathbf{b}$ so that $\mathbf{a}=\mathbf{b}^{\prime}=\mathbf{b} \bigvee \mathbf{0}^{\prime}$ $\& \mathbf{b} \mid \mathbf{0}^{\prime}$. Choose $K$ so that $\mathbf{d}(K)=\mathbf{b}$. Then, $\left.\mathbf{d}(K) \bigvee \mathbf{0}^{\prime}=\mathbf{d}\left(7^{\prime}\right)_{K}\right)=\mathbf{d}(K)^{\prime}$.

3. By Theorem 4(3) and Corollary 3 , if $K \in \Sigma_{1}$ and $K$ is infinite, then $\left.\mathbf{d}\left({ }^{2}\right)_{K}\right)$ =1. By a theorem of Sack's [6, p. 107],

$$
\exists K\left[K \in \Sigma_{1} \& 0<\mathbf{d}(K)<1 \& \mathbf{d}(K)^{\prime}=2\right] .
$$

Thus, $\exists K\left[\mathbf{d}(K)<\mathbf{d}\left(7_{K}\right)<\mathbf{d}(K)^{\prime}\right]$.

3. Relative recursiveness. In the introduction to this paper we asked whether $\mathbf{d}\left(6 \theta_{K}\right)$ is a function of $\mathbf{d}(K)$. In this section we show that $A \leq_{r} B$ does not imply $W_{A} \leq_{r} 60_{B}$, and, more strongly, show that $\mathrm{d}\left(W_{K}\right)$ is rot a function of $\mathrm{d}(K)$. We then show (see Corollary 8 and Theorem 10) that for each degree $\mathbf{d}$ there exist sets $A$ and $B$ so that $\mathrm{d}(A)=\mathrm{d}(B)=\mathrm{d}, \mathrm{d}\left(W_{A}\right)=\mathrm{d}(A)^{\prime}$ and $\mathrm{d}\left(6_{B}\right)=\mathrm{d}(B) \bigvee 1$.

Define $p(x, y)=(x+y)^{2}+y$. Define $t(x)=n$, where $n^{2}$ is the largest square less than $x$. Define $s(x)=x-t(x)^{2}$ and $u(x)=t(x)-s(x), x \neq 3$. Then $u(p(x, y))$ $=x$ and $s(p(x, y))=y$.

It follows from Definition 4 and Lemma 2 that $p(x, y) \in \operatorname{Spr}_{2}$. Thus, $p(x, y)$ has an $\omega$-representation $\phi\left(M^{1}, N^{1}, F^{1}, \ldots\right)$ so that

$$
\operatorname{rng} p(x, y)=S\left(\phi\left(M^{1}, N^{1}, F^{1}, \ldots\right) \wedge \forall x F^{1} x\right) \text {. }
$$


Let $\sigma(x)$ be the number theoretic function defined by

$$
\left.\sigma(n)=\Gamma_{\exists !} x M^{1} x \wedge \phi\left(M^{1}, N^{1}, F^{1}, \ldots\right) \wedge \forall x F^{1} x\right\urcorner .
$$

We have now the following lemma.

Lemma 3. $R(\sigma(n), k) \equiv \exists y\left[k=(n+y)^{2}+y\right]$.

Theorem 7. $\forall A \quad \exists B\left[B \leq_{r} A \& \mathcal{B}_{B}=A^{\prime}\right]$.

Proof. Let a set $A$ be given. Choose $\exists y S^{A}(x, y)$ to be a complete $A$-generable predicate. By Lemma 3 and the definitions preceding Lemma 3,

$$
\exists y S^{A}(x, y) \equiv \exists y\left[R(\sigma(x), y) \& S^{A}(u(y), s(y))\right] .
$$

Define $B=\left\{y: S^{A}(u(y), s(y))\right\} . B \leq_{r} A . \quad \exists y S^{A}(x, y) \equiv \exists y[R(\sigma(x), y) \& y \in B]$. Thus, $\exists y S^{A}(x, y) \leq_{m} W_{B}$. $W_{B}$ r.e. $A$ follows from Theorem $4(1)$, since $B \leq_{r} A$. Thus, $W_{B}$ is complete for $A$.

Corollary 5. $\exists A, B\left[B \leq_{r} A \& \mathbb{C}_{A} \Varangle_{r} C_{B}\right]$.

Proof. Choose $A$ so that $\mathbf{d}(A)=\mathbf{d}\left({ }^{\circ} \theta_{A}\right)$. Then apply Theorem 7 .

Corollary 6. $\left.\exists K\left[\mathbf{d}\left(60_{K}\right)=\mathbf{d}(K)^{\prime} \& \mathbf{d}(6)_{K}\right)=\mathbf{d}(K)\right]$.

Proof. Choose ${ }^{\exists} y \forall z P(x, y, z)$ to be a complete $\Sigma_{2}$ predicate. $\exists y \forall z P(x, y, z) \equiv$ $\exists y[R(\sigma(x), y) \& \forall z P(u(y), s(y), z)]$. Let $K=\{y: \forall z P(u(y), s(y), z)\}$. Then, $\exists y \forall z P(x, y, z) \leq_{m} W_{K}$. But, $W_{K} \in \Sigma_{2}$. Thus, $\mathbf{d}\left(W_{K}\right)=0 "$. By Theorem $4(3)$ and Corollary $3(2)$, since $K$ is the complement of an r.e. set, $\mathbf{d}\left(7_{\bar{K}}\right)=\mathbf{0}^{\prime}$.

Thus, $60_{K}$ does not induce a function on degrees and $6 b_{K}$ does not preserve relative recursiveness.

Corollary 7. (1) $\left.\exists K\left[\mathbf{d}\left(\Re_{K}\right)=\mathbf{d}(K) \& \mathbf{d}()_{K}\right)=\mathbf{d}(K)^{\prime}\right]$.

(2) $\exists K\left[\mathbf{d}\left(\oslash_{K}\right)=\mathbf{d}(K)^{\prime} \& \mathbf{d}\left(\bigodot_{K}^{-}\right)=\mathbf{d}(K)\right]$.

Proof. Corollary 6 and Lemma 1.

Thus, the functions $M$ and $\mathcal{O}$ also do not induce functions on the degrees, and therefore do not preserve relative recursiveness.

Definition 5. Let $\phi$ be a formula in prenex normal form and $M^{1}$ a one place predicate letter, not occurring in $\phi$. Define $\phi_{M^{1}}, \phi$ relativized to $M^{1}$, as follows:

(i) If $\phi$ is quantifier free and contains occurrences of the variables $x_{0}, \ldots$, $x_{n-1}$ and no others, then $\phi_{M^{1}}$ is $\phi \wedge M^{1}\left(x_{0}\right) \wedge \ldots \wedge M^{1}\left(x_{n-1}\right)$;

(ii) if $\phi$ is $\exists y \psi, \phi_{M^{1}}$ is $\exists y\left[M^{1}(y) \wedge \psi_{M^{1}}\right]$;

(iii) if $\phi$ is $\forall y \psi, \phi_{M^{1}}$ is $\forall y\left[M^{1}(y) \rightarrow M_{M}^{1}\right]$.

$A_{n}$ easy argument proves the following lemma. 
Lemma 4. For every formula $\phi, \phi$ bas a model of finite cardinality $y$ if and only if $\phi_{M^{1}}$ has a model il so that $\left\|M^{1}\right\|=y$.

Lemma 5. For every function $f$ belonging to $\mathrm{Spr}_{1}$ there is a recursive function $g$ so that

$$
\forall x \forall y[R(x, y) \rightarrow R(g(x), f(y))]
$$

and

$$
\forall x \forall z \exists y[R(g(x), z) \rightarrow(z=f(y) \& R(x, y))]
$$

Proof. Assume $f \in \mathrm{Spr}_{1}$. By Definition $4, f$ has an $\omega$-representation $\phi\left(M^{1}, F^{1}, \ldots\right)$ so that $\left[\phi\left(M^{1}, F^{1}, \ldots\right) \& \forall x F^{1}(x)\right]$ has a model of cardinality $z$ if and only if $\exists y[z=f(y)]$.

Let $g(x)=\left\ulcorner_{\psi_{M} 1} \wedge \phi \wedge \forall x F^{1} x^{\top}\right.$, where $\psi=\left\llcorner^{x}\right.$, , and suppose $R(x, y) . \psi$ has a model of cardinality $y$. Thus, by Lemma $4, \psi_{M^{1}}$ has a model $\mathcal{U}$ so that $\left\|M_{\mathscr{q}}^{1}\right\|=y$. Since $f(y)>y$, थ can be extended and expanded to a model of $\psi_{M_{1}} \wedge$ $\phi\left(M^{1}, F^{1}, \ldots\right) \wedge \forall x F^{1} x$ of cardinality $f(y)$. Thus $R(g(x), f(y))$.

Suppose $R(g(x), z)$. Then, $\psi_{M^{1}} \wedge \phi\left(M^{1}, F^{1}, \ldots\right) \wedge \forall x F^{1} x$ has a model \& of cardinality $z$. The restriction to $M_{\mathfrak{Q}}^{1}$ is a model of cardinality $y$ of $\psi$, where $f(y)=z$. Thus $R(x, y)$.

Definition 6. $A \leq_{m s} B \leftrightarrow A \leq_{m} B$ by a function $f \in \mathrm{Spr}_{1}$.

$\leq_{m s}$ is a reducibility. That is, $\leq_{m s}$ is a reflexive and transitive subrelation of $\leq_{r}$. In fact, if $A \leq_{m} B$ by $f \in \mathrm{Spr}_{1}$, and $B \leq_{m} C$ by $g \in \mathrm{Spr}_{1}$, then $x \in A \leftrightarrow$ $g(f(x)) \in C$. Therefore, by Lemma $2(2), A \leq_{m s} C$. Hence $\leq_{m s}$ is transitive. Since the identity function belongs to $\mathrm{Spr}_{1}, \leq_{m s}$ is reflexive.

Theorem 8. If $A \leq_{m s} B$, then $60_{A} \leq_{m}$ i ${ }_{B}$.

Proof. Suppose $A \leq_{m} B$ by $f \in \mathrm{Spr}_{1}$. By Lemma 5, there exists a recursive function $g$ so that

$$
R(x, y) \rightarrow R(g(x), f(y))
$$

and

$$
\begin{gathered}
\forall x, z \exists y[R(g(x), z) \rightarrow(z=f(y) \& R(x, y))] . \\
x \in \mathbb{U}_{A} \rightarrow \exists y[R(x, y) \& y \in A] \rightarrow \exists y[R(g(x), f(y)) \& y \in A] \\
\rightarrow \exists y[R(g(x), f(y)) \& f(y) \in B] \rightarrow \exists y[R(g(x), y) \& y \in B] \rightarrow g(x) \in \mathbb{C}_{B} . \\
g(x) \in \hat{\mathbb{A}}_{B} \rightarrow \exists z[R(g(x), z) \& z \in B] \\
\rightarrow \exists y[R(g(x), f(y)) \& f(y) \in B] \rightarrow \exists y[R(x, y) \& y \in A] \rightarrow x \in \mathbb{C}_{A}
\end{gathered}
$$


Thus $W_{A} \leq_{m} W_{B}$ by $g$, concluding the proof of Theorem 8 .

Define the recursive sup of the two sets $A$ and $B$ by

$$
2 x \in A \bigvee B \leftrightarrow x \in A, \quad 2 x+1 \in A \bigvee B \leftrightarrow x \in B
$$

It is clear that $A \leq_{m} A \vee B, B \leq_{m} A \vee B$, and that $\mathrm{d}(A \vee B)$ is the least upper bound of $\mathrm{d}(A)$ and $\mathrm{d}(B)$.

Lemma 6. For any two sets $A$ and $B, W_{A} \leq_{m} W_{A \vee B}$ and $6 D_{B} \leq_{m} W_{A \vee B}$.

Proof. By Lemma 2, the functions $2 x$ and $2 x+1$ belong to $\operatorname{Spr}_{1}$.

The proof follows then from Theorem 8.

Theorem 9. $\forall A \quad \exists C\left[\mathrm{~d}(C)=\mathbf{d}(A) \& \mathcal{W}_{C}\right.$ is complete A-generable $]$.

Proof. By Theorem $7, \exists B\left[B \leq_{r} A \& B_{B}\right.$ is complete for $\left.A\right]$. Let $C=A \vee B$. $B \leq_{r} A$, thus $\mathrm{d}(C)=\mathrm{d}(A)$. $W_{C}$ is r.e. in $C$ and $C \leq_{r} A$, thus $W_{C}$ is r.e. in $A$. By Lemma $7, W_{B} \leq_{m} 6 O_{C}$; also $W_{B}$ is complete for $A$. Thus, $W_{C}$ is complete for $A$.

Corollary 8. $\forall \mathrm{d} \exists A\left[\mathrm{~d}(A)=\mathrm{d} \& \mathrm{~d}\left(6_{A}\right)=\mathrm{d}(A)^{\prime}=\mathbf{d}^{\prime}\right]$.

The following theorem (obtained by Thomas Grilliot, in personal communication) gives a positive solution to a question raised in [8].

Theorem 10. $\forall \mathbf{d} \exists A\left[\mathbf{d}(A)=\mathbf{d} \& \mathbf{d}\left(C_{A}\right)=\mathbf{d} \vee 1\right]$.

Proof. By Corollary 3, we already have this result for the case $\mathbf{d}=\mathbf{0}$. Therefore, assume that $\mathbf{d}>\mathbf{0}$, and choose $K$ so that $\mathrm{d}(K)=\mathrm{d}$. Let $\mathrm{Ch}_{K}(n)$ denote the characteristic function of $K$, and let $\widetilde{C h}_{K}(n)\left(=\Pi_{i<n} P_{i}^{C_{h} K^{(i)}}\right.$, see [5, p. 231]) be the course-of-values function for $\mathrm{Ch}_{K}(n)$. Then, define $A$ to be the complement of $\left\{\widetilde{C h}_{K}(n): n \in \omega\right\} . K \leq_{r} A$, and $A \leq_{r} K$. Also, it is easy to see that $\bar{A}$ is recursive in every infinite subset of $\bar{A}$.

By Corollary 4 , it suffices to show that $\mathrm{d}\left(\mathbb{C O}_{A}\right) \leq \mathrm{d}(A) \bigvee 1$. Let $\phi$ be any formula of $\mathcal{\varrho}$. Since $\mathrm{d}(A)>0, A$ is not recursive in $S(\phi)$. Therefore $S(\phi)$ cannot be an infinite subset of $\bar{A}$. Hence, either $S(\phi)$ is finite, or $\ulcorner\phi\urcorner \in \mathcal{U}_{A}$. That is, either $\exists y \forall z>y \bar{R}(\ulcorner\phi\urcorner, y)$ or $\left.\exists y\left[\bar{R}\left(\Gamma_{\phi}\right\urcorner, y\right) \& y \in A\right]$. The function $f(x)=$ $\mu_{y}[[R(x, y) \& y \in A] \bigvee \forall z>y \bar{R}(x, z)]$ is recursive in $A$ and $\mathbf{0}^{\prime}$, and

$$
\left.\left.\left.r_{\phi}\right\urcorner \in \mathbb{C}_{A} \leftrightarrow \exists y \leq f\left(r_{\phi}\right\urcorner\right)\left[R\left(r_{\phi}\right\urcorner, y\right) \& y \in A\right] .
$$

Hence, $\mathrm{d}\left(\mathrm{CO}_{A}\right) \leq \mathrm{d}(A) \bigvee 1$.

4. Values of $\mathrm{d}\left(\left(_{K}\right)\right.$, for $K$ of a given degree. Are $\mathrm{d}(K) \bigvee 0^{\prime}$ or $\mathrm{d}(K)^{\prime}$ the only possible values for $\mathrm{d}\left(\hat{W}_{K}\right)$ for any $K$ ? In this section we describe the extent to which $d\left(\mathbb{W}_{K}\right)$ is independent from $d(K)$, within the bounds given by Theorem 4 and Corollary 4 . 
Lemma 7. There is a recursive function $f$ so that $R(x, 2 y) \leftrightarrow R(f(x), y)$.

Proof. Let $x_{0}, x_{1}, \ldots$, be a complete list of the individual variables in $\mathcal{L}$. Let $S$ be a binary predicate letter and let $a$ and $b$ be individual constant letters. Given a formula $\phi$ in $\mathcal{L}$, let $x_{k}$ be the highest index variable which occurs in $\phi$. Let $u_{i}$ denote the variable $x_{k+1+i}$. Then, none of the variables $u_{0}, u_{1}, \ldots$, occurs in $\phi$. Also, we may suppose without loss of generality that $\phi$ contains no occurrences of $S, a$ and $b$. (Otherwise, $\phi_{1}$ can be found uniformly, where $\phi_{1}$ contains no occurrences of $S, a$ and $b$, and $\left.\left.R\left(\Gamma_{\phi}\right\urcorner, 2 y\right) \leftrightarrow R\left(\Gamma_{\phi_{1}}\right\urcorner, 2 y\right)$.) We define a new formula $\phi^{*}$ as follows:

$$
\begin{gathered}
\left(x_{i}=x_{j}\right)^{*} \text { is } x_{i}-x_{j} \wedge u_{i}=u_{j} ; \\
P^{n}\left(x_{i_{1}}, \ldots, x_{i_{n}}\right)^{*} \text { is } P^{2 n}\left(x_{i_{1}}, u_{i_{1}}, \cdots, x_{i_{n}}, u_{i_{n}}\right) ; \\
\left(\psi_{1} \wedge \psi_{2}\right)^{*} \text { is } \psi_{1}^{*} \wedge \psi_{2}^{*} ; \\
\left.(\urcorner \psi)^{*} \text { is }\right\urcorner\left(\psi^{*}\right) ; \\
\left(\exists x_{i} \psi\right)^{*} \text { is } \exists x_{i} \exists u_{i}\left[S\left(x_{i}, u_{i}\right) \wedge \psi^{*}\right] .
\end{gathered}
$$

Detine

$$
\begin{aligned}
& T(\phi)=\phi^{*} \wedge a \neq b \wedge[ \forall x S(x, a) \wedge \forall x S(x, b) \\
& \wedge \forall x \forall y(S(x, y) \rightarrow y=a \bigvee y=b) \wedge د\left(x_{i_{1}}, u_{i_{1}}\right) \wedge \cdots \\
& \wedge S\left(x_{\left.\left.i_{n}, u_{i_{n}}\right)\right],}\right.
\end{aligned}
$$

where $x_{i_{1}}, \cdots, x_{i_{n}}$ is a list of the free variables occurring in $\phi$.

Claim. $\phi$ is satisfiable in a structure of cardinality $2 y$ if and only if $T(\phi)$ is satisfiable in a structure of cardinality $y$.

Proof. We first show that if $\phi$ is satisfiable in a structure of cardinality $2 y$, then $T(\phi)$ is satisfiable in a structure of cardinality $y$. If a formula $\phi$ holds in a structure of cardinality $2 y$, then let

$$
A=\{\langle 1,1\rangle,\langle 2,1\rangle, \cdots,\langle y, 1\rangle,\langle 1,2\rangle,\langle 2,2\rangle, \cdots,\langle y, 2\rangle\}
$$

be the domain of such a structure, $\mathscr{A}$. Define a structure $B$ with domain $B=\{1,2$, $\cdots, y\}$ as follows: by

(1) If $R_{\boldsymbol{A}}$ is a $k$-ary relation on $A$, then $R_{\boldsymbol{B}}$ is a $2 k$-ary relation on $B$ defined

$$
R_{\boldsymbol{g}}\left(i_{1}, j_{1}, \ldots, i_{k}, j_{k}\right) \leftrightarrow R_{\mathfrak{q}}\left(\left\langle i_{1}, j_{1}\right\rangle, \ldots,\left\langle i_{k}, j_{k}\right\rangle\right),
$$

for $i_{1}, \cdots, i_{k} \in\{1,2, \ldots, y\}$, and $j_{1}, \ldots, j_{k} \in\{1,2\}$. 
(2) $S_{\forall}=\{\langle i, j\rangle: i=1, \cdots, y \&(j=1$ or $j=2)\}$.

(3) $a$ is $1, b$ is 2 .

It is clear that $[\forall x S(x, a) \wedge \forall x S(x, b) \wedge \forall x \forall y(S(x, y) \rightarrow y=a \bigvee y=b)]$ holds in $B$.

We prove by induction that $\phi$ is satisfiable in $\mathcal{U}$ (by an assignment $\alpha$ ) if and only if $T(\phi)$ is satisfiable in $B$ (by an assignment $\beta$ ). Moreover, $\alpha\left(x_{i}\right)=$ $\left\langle\beta\left(x_{i}\right), \beta\left(u_{i}\right)\right\rangle$.

Case $\phi$ is $x_{i}=x_{j}$. If $\alpha$ satisfies $\phi$ in $\mathscr{U}$, then for some $\langle s, t\rangle \in A, \alpha\left(x_{i}\right)=$ $\alpha\left(x_{j}\right)=\langle s, t\rangle$. Define $\beta$ by $\beta\left(x_{i}\right)=\beta\left(x_{j}\right)=s$ and $\beta\left(u_{i}\right)=\beta\left(u_{j}\right)=t$. Then $\beta$ satisfies $\phi^{*}$ in $\mathbb{B}$. Since $t=1$ or $t=2, \beta$ satisfies $T(\phi)$ in $\mathbb{B}$.

$\phi^{*}$ is $x_{i}=x_{j} \wedge u_{i}=u_{j}$. If $\beta$ satisfies $T(\phi)$ in $B$, then $\beta\left(x_{i}\right)=\beta\left(x_{j}\right)=s$ and $\beta\left(u_{i}\right)=\beta\left(u_{j}\right)=t$, for $s, t \in B$. Also, $S_{\mathfrak{B}}(s, t)$. Thus, $\langle s, t\rangle \in A$. Define $a$ by $\alpha\left(x_{i}\right)=\alpha\left(x_{j}\right)=\langle s, t\rangle$. a satisfies $\phi$ in $\mathcal{U}$.

Case $\phi$ is $P\left(x_{1}, \cdots, x_{n}\right)$. If there is an assignment $\alpha$ so that $P_{\mathfrak{Q}}\left(\alpha\left(x_{1}\right), \ldots, \alpha\left(x_{n}\right)\right)$, define $\beta\left(x_{i}\right)$ and $\beta\left(u_{i}\right), i=1, \ldots, n$, so that $\alpha\left(x_{i}\right)=$ $\left\langle\beta\left(x_{i}\right), \beta\left(u_{i}\right)\right\rangle$. Then, by definition of $P_{\mathfrak{B}}, P_{\mathfrak{B}}\left(\beta\left(x_{1}\right), \beta\left(u_{1}\right), \ldots, \beta\left(x_{n}\right), \beta\left(u_{n}\right)\right)$. That is, $\beta$ satisfies $\phi^{*}$ in $\mathscr{B}$. Therefore, $\beta$ satisfies $T(\phi)$ in $B$.

Suppose $\beta$ satisfies $T(\phi)$ in $B . \phi^{*}$ is $P\left(x_{1}, u_{1}, \ldots, x_{n}, u_{n}\right)$. $P_{\mathfrak{B}}\left(\beta\left(x_{1}\right), \beta\left(u_{1}\right), \ldots, \beta\left(x_{n}\right), \beta\left(u_{n}\right)\right)$, and $S_{\mathfrak{g}}\left(\beta\left(x_{i}\right), \beta\left(u_{i}\right)\right), i=1, \cdots, n$. Thus $\left\langle\beta\left(x_{i}\right), \beta\left(u_{i}\right)\right\rangle \in A, i=1, \cdots, n$. Define $\alpha\left(x_{i}\right)=\left\langle\beta\left(x_{i}\right), \beta\left(u_{i}\right)\right\rangle$. a satisfies $\phi$ in $\mathcal{U}$.

Case $\phi$ is $\psi_{1} \wedge \psi_{2}$. If a satisfies both $\psi_{1}$ and $\psi_{2}$ in $\mathcal{U}$, then by induction hypothesis $\beta$ satisfies $\psi_{1}^{*}$ in $B$ and $\beta$ satisfies $\psi_{2}^{*}$ in $B$, where $\beta$ is defined so that $\alpha\left(x_{i}\right)=\left\langle\beta\left(x_{i}\right), \beta\left(u_{i}\right)\right\rangle$, for each $i$. Thus $\beta$ satisfies $\phi^{*}=\psi_{1}^{*} \wedge \psi_{2}^{*}$ in $B$. Thus, $\beta$ satisfies $T(\phi)$ in $B$.

The other direction is identical. The case $\phi$ is $\urcorner \psi$ is straightforward.

Case $\phi$ is $\exists x_{i} \psi$. If $\exists x_{i} \psi$ is satisfied in $\mathcal{U}$, then some assignment $\alpha$ satisfies $\psi\left(x_{i}\right)$ in 2 . Thus $\beta$ defined by $\alpha\left(x_{j}\right)=\left\langle\beta\left(x_{j}\right), \beta\left(u_{j}\right)\right\rangle$, for all $j$, satisfies $\psi^{*}\left(x_{i}, u_{i}\right)$ in $B$. Also, $S_{\mathscr{B}}\left(\beta\left(x_{i}\right), \beta\left(u_{i}\right)\right)$. Hence $\beta$ satisfies $\dot{\exists} x_{i}, u_{i}\left[S\left(x_{i}, u_{i}\right) \wedge \psi^{*}\right]$ in $B$. Thus, $\beta$ satisfies $T(\phi)$ in $B$.

The other direction is similar.

We have shown that if $\phi$ is satisfiable in a structure of cardinality $2 y$, then $T(\phi)$ is satisfiable in a structure of cardinality $y$. We show now that if $T(\phi)$ is satisfiable in a structure of cardinality $y$, then $\phi$ is satisfiable in a structure of cardinality $2 y$.

If $T(\phi)$ is satisfiable in a structure $(\mathcal{S}$ of cardinality $y$, we may assume that $C=\{1,2, \ldots, y\}$ is the domain, $a$ is $1, b$ is 2 , and $S_{\mathfrak{S}}(i, j) \leftrightarrow j=1$ or $j=2$.

Define a structure $B$ from $(S$ as follows:

(1) $B=\{1, \ldots, y\}$, the domain of $B$;

(2) $S_{\mathfrak{B}}=S_{\mathfrak{C}}$; 
(3) $a$ is $1, b$ is 2 ;

(4) $P_{\mathfrak{B}}\left(i_{1}, j_{1}, \ldots, i_{n}, j_{n}\right) \leftrightarrow P_{\mathfrak{S}}\left(i_{1}, j_{1}, \ldots, i_{n}, j_{n}\right) \wedge S_{\mathfrak{B}}\left(i_{1}, j_{1}\right) \wedge \ldots \wedge$ $S_{\mathfrak{B}}\left(i_{n}, j_{n}\right)$. (Note that only $2 k$-ary relations appear in $T(\phi)$.)

We show by induction that every assignment $\gamma$ which satisfies $T(\phi)$ in (S) also satisfies $T(\phi)$ in $\mathbb{B}$, and every assignment $\beta$ which satisfies $T(\phi)$ in $\mathbb{B}$ also satisfies $T(\phi)$ in $(5$.

Our result follows easily from this, because $B$ is obtainable from a structure Q 0 of cardinality $2 y$ as in the previous part of the proof, and we know that $T(\phi)$ is satisfiable in $B$ if and only if $\phi$ is satisfiable in $\mathcal{U}$. domain.

If $\phi$ is $x_{i}=x_{j}$, there is nothing to show, since $B$ and $(S$ have the same

Case $\phi$ is $P\left(x_{1}, \cdots, x_{n}\right)$. Assume $T(\phi)$ is satisfiable in $\mathcal{S}$. Then there is a $2 n$-ary relation $P_{\mathfrak{S}}$ on $\{1, \ldots, y\}$ and an assignment $\gamma$ to $(S)$ so that $P_{\mathfrak{G}}\left(\gamma\left(x_{1}\right), \gamma\left(u_{1}\right), \cdots, \gamma\left(x_{n}\right), \gamma\left(u_{n}\right)\right)$, and so that $S_{\mathfrak{S}}\left(\gamma\left(x_{i}\right), \gamma\left(u_{i}\right)\right), i=1, \cdots, n$. Thus

$$
P_{\mathfrak{B}}\left(\gamma\left(x_{1}\right), \gamma\left(u_{1}\right), \cdots, \gamma\left(x_{n}\right), \gamma\left(u_{n}\right)\right) \text {. }
$$

That is, $\gamma$ satisfies $T(\phi)$ in $B$.

It is obvious that an assignment satisfying $T(\phi)$ in $B$ also satisfies $T(\phi)$ in $(5$. This direction is clear in the following cases too.

Case $\phi$ is $\psi_{1} \wedge \psi_{2}$. Suppose an assignment $\gamma$ satisfies $T(\phi)$ in (S. $S_{\mathfrak{G}}\left(\gamma\left(x_{i}\right), \gamma\left(u_{i}\right)\right)$ for all free variables $x_{i}$ occurring in $\phi$. Thus $\gamma$ satisfies $\psi_{1}^{*}$ and $\psi_{2}^{*}$ in $B$, and $\gamma$ satisfies $T(\phi)$ in $B$.

Case $\phi$ is $7 \psi$. Suppose $T(7 /)$ is satisfied by an assignment $\gamma$ in (S. $S_{5}\left(\gamma\left(x_{i}\right), \gamma\left(u_{i}\right)\right)$ for all free variables $x_{i}$ occurring in $\psi$. Thus, as above, $\gamma$ is an assignment to B. $\gamma$ satisfies $7\left(\psi^{*}\right)$ in (S. Thus, $\gamma$ does not satisfy $\psi^{*}$ in (S. By induction hypothesis, $\gamma$ does not satisfy $\psi^{*}$ in $\mathscr{R}$. Thus $\gamma$ satisfies $7\left(\psi^{*}\right)=\left(7 \psi^{*}\right)$ in $\mathbb{B}$. That is, $T(\phi)$ is satisfied by $\gamma$ in $\mathbb{B}$.

Case $\phi$ is $\exists x_{i} \psi$. If $T(\phi)$ is satisfied in (, , then $\psi^{*}$ is satisfied by some $\gamma$ in $\left(S . S\left(x_{i}, u_{i}\right)\right.$. Thus $\gamma$ is an assignment to $B$ and $\psi *\left(x_{i}, u_{i}\right)$ is satisfied by $\gamma$ in $B$. Thus $T(\phi)$ is satisfied by $\gamma$ in $B$.

The proof of the claim is complete. Let $\mathcal{C}(\phi)$ denote the universal closure of $\phi . \phi$ is valid in a structure $\mathcal{Q}$ of cardinality $2 y$ if and only if $\mathcal{Q}(\phi)$ is satisfiable in $\mathcal{Q}$ if and only if $T(\mathcal{Q}(\phi))$ is satisfiable in a structure $B$ of cardinality $y$ if and only if $T(\mathcal{Q}(\phi))$ is valid in $\mathfrak{B}$ (since $T(\mathcal{Q}(\phi))$ is closed). Define $f(x)=$ $\left\ulcorner_{T}(\mathcal{C l}(\phi))\right.$, for $x=\left\ulcorner_{\phi}\right\urcorner$. Then, $R(x, 2 y) \rightarrow R(f(x), y)$.

Lemma 8. There is a recursive function $g$ so that $R(x, 2 y+1) \leftrightarrow R(g(x), y)$.

Proof. As in the proof of Lemma 7, given $\phi$ in $\mathcal{\varrho}$, let $x_{k}$ be the highest index variable which occurs in $\phi$ and let $u_{i}$ denote the variable $x_{k+1+i}$, all $i$. Again as in the proof of Lemma 7 , we can suppose without loss of generality that $\phi$ con- 
tains no occurrences of the binary predicate letter $S$ and $\phi$ contains no occurrences of the individual constant letters $a, b$, and $c$. The formula $\phi^{*}$ is defined for $\phi$ as in the previous proof. Define

$$
\begin{aligned}
& T(\phi)=\phi^{*} \wedge a \neq b \wedge a \neq c \wedge b \neq c \\
& \wedge[\forall x S(x, a) \wedge \forall x S(x, b) \wedge S(c, c) \\
& \wedge \forall x \forall y(S(x, y) \rightarrow(y=a \bigvee y=b \bigvee(x=c \wedge y=c))) \\
& \left.\wedge S\left(x_{i_{1}}, u_{i}\right) \wedge \cdots \wedge S\left(x_{i_{n}}, u_{i_{n}}\right)\right],
\end{aligned}
$$

where $x_{i_{1}}, \cdots, x_{i_{n}}$ is a list of the distinct free variables occurring in $\phi$.

Claim. $\phi$ is satisfiable in a structure of cardinality $2 y+1$ if and only if $T(\phi)$ is satisfiable in a structure of cardinality $y$.

If $\phi$ holds in a structure of cardinality $2 y+1$, then let $A=\{\langle 1,1\rangle, \ldots,\langle y, 1\rangle$, $\langle 1,2\rangle, \ldots,\langle y, 2\rangle,\langle 3,3\rangle\}$ be the domain of such a structure, $\overrightarrow{\mathrm{l}}$. Define a structure $B$ with domain $B=\{1, \ldots, y\}$ as follows:

(1) If $R_{\mathscr{q}}$ is a $k$-ary relation on $A$, then $R_{\mathscr{B}}$ is a $2 k$-ary relation on $B$ defined by

$$
R_{\mathfrak{B}}\left(i_{1}, j_{1}, \cdots, i_{k}, j_{k}\right) \leftrightarrow R_{\mathfrak{g}}\left(\left\langle i_{1}, j_{1}\right\rangle, \ldots,\left\langle i_{k}, j_{k}\right\rangle\right),
$$

for $i_{1}, \ldots, i_{k} \in\{1, \ldots, y\} ; j_{1}, \ldots, j_{k} \in\{1,2,3\}$.

(2) $S_{\mathfrak{B}}$ is a binary relation defined by $S_{\mathfrak{B}}(i, 1)$ and $S_{\mathfrak{B}}(i, 2)$ for all $i=1,2$, $\cdots, y$, and $S_{\boldsymbol{B}}(3,3)$.

(3) $a$ is $1, b$ is 2 , and $c$ is 3 .

An induction argument shows that $\phi$ is satisfied in $\mathcal{U}$ (by an assignment $\alpha$ ) if and only if $T(\phi)$ is satisfied in $B$ (by an assignment $\beta$ ). Moreover, for all $i$, $\alpha\left(x_{i}\right)=\left\langle\beta\left(x_{i}\right), \beta\left(u_{i}\right)\right\rangle$. It follows that if $\phi$ is satisfiable in a structure of cardinality $2 y+1$, then $T(\phi)$ is satisfiable in a structure of cardinality $y$.

Conversely, if $T(\phi)$ is satisfiable in a structure $(5)$ of cardinality $y$, we may assume that $C=\{1, \ldots, y\}$ is the domain, $a$ is $1, b$ is $2, c$ is 3 , and $S_{\mathfrak{G}}(i, j) \leftrightarrow$ $(j=1$ or $j=2$ or $(i=3 \& j=3))$. Define a structure $B$ from $\subseteq$ as follows:

(1) $B=\{1, \ldots, y\}$

(2) $S_{\mathfrak{B}}=S_{\mathbb{S}}$;

(3) $a$ is $1, b$ is $2, c$ is 3 ;

(4) $P_{\mathfrak{B}}\left(i_{1}, j_{1}, \ldots, i_{n}, j_{n}\right) \leftrightarrow P_{\mathfrak{G}}\left(i_{1}, j_{1}, \ldots, i_{n}, j_{n}\right) \wedge S_{\mathfrak{s}}\left(i_{1}, j_{1}\right) \wedge \ldots \wedge S_{\mathfrak{G}}\left(i_{n}, j_{n}\right)$.

As in the proof of Lemma 7, an induction argument shows that if $T(\phi)$ is satisfied in $(S$ by an assignment $\gamma$, then $T(\phi)$ is satisfied in $B$ by $\gamma$ and conversely.

$B$ is obtainable from a structure $\mathcal{U}$ of cardinality $2 y+1$ as in the previous part of the proof; and we know that $T(\phi)$ is satisfiable in $B$ if and only if $\phi$ is satisfiable in $\mathscr{l}$. Thus, if $T(\phi)$ is satisfiable in a structure of cardinality $y$, then 
$\phi$ is satisfiable in a structure of cardinality $2 y+1$. This completes the proof of the claim.

Define $g(x)=\left\ulcorner_{T}(\mathcal{Q}(\phi))\right\urcorner$, for $x=\ulcorner\phi\urcorner$. Then $R(x, 2 y+1) \leftrightarrow R(g(x, y))$.

Theorem 11. $\mathrm{d}\left(68_{A \vee B}\right)=\mathrm{d}\left(66_{A}\right) \vee \mathrm{d}\left(6 \mathrm{CO}_{B}\right)$.

Proof. By Lemma $6, \mathrm{~d}\left(60_{A}\right) \vee \mathrm{d}\left(6_{B}\right) \leq \mathrm{d}\left(6_{A \vee B}\right)$.

$$
\begin{aligned}
\exists y[R(x, y) \& y \in A \bigvee B] \leftrightarrow & \exists y[R(x, 2 y) \& 2 y \in A \bigvee B] \\
& \bigvee \exists y[R(x, 2 y+1) \& 2 y+1 \in A \bigvee B] \\
\leftrightarrow & \exists y[R(x, 2 y) \& y \in A] \bigvee \exists y[R(x, 2 y+1) \& y \in B]
\end{aligned}
$$

By Lemmas 7 and 8 , let $f$ satisfy $R(x, 2 y) \rightarrow R(f(x), y)$ and let $g$ satisfy $R(x, 2 y+1) \leftrightarrow R(g(x), y)$. Then,

$$
\exists y[R(x, 2 y) \& y \in A] \leftrightarrow \exists y[R(f(x), y) \& y \in A]
$$

and

$$
\exists y[R(x, 2 y+1) \& y \in B] \leftrightarrow \exists y[R(g(x), y) \& y \in B]
$$

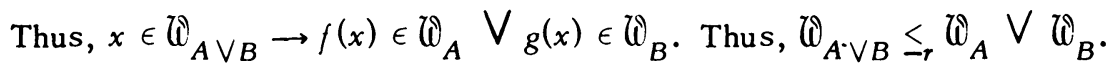

We are now ready to prove our main results.

Theorem 12. $\forall \mathbf{a} \geq \mathbf{0}^{\prime} \forall \mathbf{b} \exists K\left[\left(\mathbf{a} \leq \mathbf{b} \leq \mathbf{a}^{\prime} \&\right.\right.$ b r.e. $\left.\left.\mathbf{a}\right) \rightarrow\left(\mathrm{d}(K)=\mathbf{a} \& \mathbf{d}\left(\mathcal{C}_{K}\right)=\mathbf{b}\right)\right]$.

Proof. (See Figure 1.) Let $\mathbf{a}$ and $\mathbf{b}$ satisfy $\mathbf{a} \geq \mathbf{0}^{\prime}$, $\mathbf{a} \leq \mathbf{b} \leq \mathbf{a}^{\prime}$, and $\mathbf{b}$ r.e. $\mathbf{a}$. By Friedberg's characterization [3], $\exists \mathbf{c}\left[\mathbf{a}=\mathbf{c}^{\prime}\right]$. b r.e. $\mathbf{c}^{\prime}$ thus by Shoenfield [9], $\exists \mathrm{d}\left[\mathbf{b}=\mathrm{d}^{\prime} \& \mathbf{c} \leq \mathbf{d} \leq \mathbf{c}^{\prime}\right]$.

By Theorem 6 , choose $A$ so that $\mathrm{d}(A)=\mathrm{d}\left(6_{A}\right)=\mathrm{a}$. By Corollary 8 , choose $B$ so that $\mathrm{d}(B)=\mathbf{d}$ and $\mathbf{d}\left(W_{B}\right)=\mathbf{d}^{\prime}=$ b. $B \leq_{r} A$. Let $K=A \bigvee B . \mathrm{d}(K)=\mathrm{d}(A)=\mathbf{a}$. By Theorem 11, $\left.\mathbf{d}\left(\mathbb{W}_{K}\right)=\mathbf{d}\left(\mathbb{W}_{A}\right)_{B}\right)=\mathbf{d}\left(\mathbb{W}_{A}\right) \vec{V}^{-r} \mathbf{d}\left(\mathbb{(}_{B}\right)=\mathbf{a} \vee \mathbf{b}=\mathbf{b}$.

$K=A \bigvee B$

$$
\mathbf{d}\left(\bar{i}_{K}\right)=\prod_{\left.\right|_{c} ^{\mathbf{d}}=\mathbf{d}(K)=\mathbf{d}^{\prime}=\mathbf{d}\left(\pi_{B}\right)}^{\mathbf{a}^{\prime}}=\mathbf{c}^{\prime}=\mathbf{d}(A)=\mathbf{d}\left(i_{A}\right)
$$

\section{Figure 1}

Theorem 13. $\forall \mathbf{a} \forall \mathbf{b} \quad \exists K\left[\mathbf{a} \leq \mathbf{b} \leq \mathbf{a}^{\prime} \rightarrow\left(\mathbf{d}(K)=\mathbf{b} \& \mathbf{d}\left(\left({ }^{8}\right)_{K}\right)=\mathbf{a}^{\prime}\right)\right]$. 
Proof. By Corollary 8 and Theorem 10 , choose sets $A$ and $B$ so that $\mathrm{d}(A)=$ $\mathbf{a}, \mathbf{d}(B)=\mathbf{b}, \mathbf{d}\left(\mathbb{C}_{A}\right)=\mathbf{a}^{\prime}$, and $\mathbf{d}\left(W_{B}\right)=\mathbf{b} \vee 1$. Let $K=A \vee B$. Then, $\mathbf{d}(K)=$ $\mathbf{d}(A \vee B)=\mathbf{d}(B)=\mathbf{b}$. By Theorem $11, \mathbf{d}\left(W_{K}\right)=\mathbf{d}\left(W_{A \vee B}\right)=\mathbf{d}\left(W_{A}\right) \vee \mathbf{d}\left(W_{B}\right)=\mathbf{a}^{\prime} \vee \mathbf{b}$ $\bigvee \mathbf{l}=\mathbf{a}^{\prime}$.

Theorem 14. $\forall \mathbf{a} \geq 0^{\prime} \forall \mathbf{b} \quad \exists K\left[\left(\mathbf{a} \leq \mathbf{b} \leq \mathbf{a}^{\prime} \&\right.\right.$ b r.e. $\left.\mathbf{a}\right) \rightarrow\left(\mathrm{d}(K) \leq \mathbf{a} \& \mathrm{~d}\left(\mathcal{G}_{K}\right)=\mathbf{a} \&\right.$ $\left.\left.\mathbf{d}(K)^{\prime}=\mathbf{b}\right)\right]$.

Proof. Using [3] and [9], as in the first paragraph of the proof of Theorem 12, $\exists \mathbf{c}, \mathbf{d}\left[\mathbf{c} \leq \mathbf{d} \leq \mathbf{c}^{\prime}=\mathbf{a} \leq \mathbf{d}^{\prime}=\mathbf{b} \leq \mathbf{a}^{\prime}\right]$. By Corollary 8 and Theorem 10 , choose $A$ and $B$ so that $\mathbf{d}(A)=\mathbf{d}, \mathbf{d}(B)=\mathbf{c}, \mathbf{d}\left(C_{A}\right)=\mathbf{d} \vee 1$, and $\mathbf{d}\left(6 B_{B}\right)=\mathbf{c}^{\prime}=\mathbf{a}$.

$$
\begin{aligned}
& \mathrm{d}(A \vee B)=\mathbf{d} \vee \mathbf{c}=\mathbf{d} \leq \mathbf{a} . \\
& \mathbf{d}(A \vee B)^{\prime}=\mathbf{d}^{\prime}=\mathbf{b} . \\
& \mathbf{d}\left(\imath_{A \vee B}\right)=\mathbf{d}\left(\imath_{A}\right) \vee \mathrm{d}\left(\imath_{B}\right)=\mathbf{d} \vee 1 \vee \mathbf{a}=\mathbf{a} .
\end{aligned}
$$

Take $K=A \bigvee B$.

\section{BIBLIOGRAPHY}

1. G. Asser, Das Represäntantenproblem in Prädikatenkalkül der ersten Stufe mit Identität, Z. Math。Logik Grundlagen Math。1 (1955), 252-263。 MR 17, 1038.

2. J. Bennett, On spectra, Doctoral Dissertation, Princeton University, Princeton, N. J., 1962 .

3. Richard Friedberg, A criterion for completeness of degrees of unsolvability, J. Symbolic Logic 22 (1957), 159-160. MR 20 \#488.

4. N. D. Jones and A. L. Selman, Turing machines and the spectra of first-order formulas with equality, Fourth ACM Sympos. on Theory of Computing, 1972, pp. 157-167.

5. S. Kleene, Introduction to metamathematics, 5th ed., Van Nostrand, Princeton, N. J., 1950.

6. Gerald E. Sacks, Degrees of unsolvability, Ann. of Math. Studies, no. 55, Princeton Univ. Press, Princeton, N. J. 1963. MR $32 \# 4013$.

7. A. L. Selman, First order formulas true in structures of arbitrary finite cardinality, Notices Amer. Math. Soc。 17 (1970), 140. Abstract \#672-202.

8. - Arithmetical reducibilities and sets of formulas valid in finite cardinality, Ph.D. Thesis, Pennsylvania State University, University Park, Pa., 1970.

9. J. R. Shoenfield, On degrees of unsolvability, Ann. of Math. (2) 69 (1959), 644653. MR $21 \# 4097$.

10. B. A. Trahtenbrot, The impossibility uj an algorithm for the decision problem for finite domains, Dokl. Akad. Nauk SSSR 70 (1950), 569-572. MR 11, 488. (Russian)

DEPARTMENT OF MATHEMATICS, PENNSYLVANIA STATE UNIVERSITY, UNIVERSITY PARK, PENNSYLVANIA 16802

Current address: Department of Mathematics, Florida State University, Tallahassee, Florida 32306 